\title{
Including immune cells in brain organoids improves model's accuracy
}

\section{BY PETER HESS}

15 NOVEMBER 2021

\section{Listen to this story:}

https://www.spectrumnews.org/wpcontent/uploads/2021/11/audio-1769513b-3294-44bb-9eec-f4833052de3c-encodings.mp3

Adding non-neuronal brain cells called microglia to cortical organoids speeds up the rate at which their neurons mature, increases the synchronization of their firing and appears to reduce cell stress, according to a new study. These changes, as well as others, help the clusters of cultured cells reflect more faithfully how the human brain develops and functions, says the team behind the work. Researchers presented the findings virtually at the 2021 Society for Neuroscience annual meeting.

Brain organoids, grown from stem cells, do not always include cells other than neurons - such as astrocytes and microglia. And in fact, the gene expression patterns of the cells in organoids do not capture those seen in human microglia, previous work shows.

"Microglia, or any immune cell population, they represent one of the major cell types that are normally absent from the organoids," says Galina Popova, a postdoctoral scholar in the lab of Tomasz Nowakowski, assistant professor of anatomy at the University of California, San Francisco.

Under normal circumstances, microglia roam the central nervous system, scanning for infections and mopping up dead and dying cells. They also prune connections among neurons, a process that seems to be disrupted in autism.

Adding microglia to brain organoids is like adding a new brick shape to a Lego set, making it possible to model the brain's micro-environment more accurately in the laboratory, Popova says. The cells could represent the "missing link" between immune activation and the resulting changes 


\section{Spectrum | Autism Research News}

https://www.spectrumnews.org

that can contribute to autism, she adds.

"As more and more attention is being paid to the neuro-immune interaction when modeling any brain development, really - early or not - it is important to take into consideration the interaction between the immune cells and other cell populations," she says.

\section{Stress relief:}

Popova and her team induced human skin cells to develop into neurons, which then self-organized into brain organoids in lab dishes. Once the organoids had developed for 15 weeks, the team added microglia extracted from fetal brain tissue.

Five weeks later, electrophysiological testing showed that the neurons in the organoids with transplanted microglia fired with significantly greater synchrony than those in the control organoids. Gene expression patterns also indicated that these neurons were maturing more rapidly.

RNA sequencing revealed some subtle transcriptional differences between the microglia-containing organoids and the controls. In radial glia, a type of immature neural cell, the presence of microglia was associated with a decrease in the expression of genes associated with viral immune responses. Radial glia in the microglia-containing organoids also showed fewer double-stranded DNA breaks, as revealed by cell staining. Taken together, these results indicate that microglia ease the amount of cell stress in the organoid environment - something that can impair cell specification, Popova says.

All of these processes associated with microglia indicate that they are crucial in creating model organoids that accurately capture the brain environment, Nowakowski says.

The team is using this model, which they described in detail in Cell Stem Cell in September, to study how microglia could protect the developing brain against specific gene mutations and environmental factors, including prenatal infections. They are also exploring how microglia responses or activation could interfere with the cells' protective role, Nowakowski says.

Read more reports from the 2021 Society for Neuroscience virtual annual meeting.

Cite this article: https://doi.org/10.53053/LMRZ5964 